\title{
Proposed Scheme for Scalable Video Broadcasting to Reduce Channel Switching Delay
}

\author{
Rohit Singh \\ Department of Computer Science and Engineering, \\ National Institute of Technology, \\ Hamirpur, India
}

\author{
Pardeep Singh \\ Department of Computer Science and Engineering, \\ National Institute of Technology, \\ Hamirpur, India
}

\begin{abstract}
Always user interaction has been one of the most crucial points when evaluating the quality of services. Mobile television is one of the most important services for the users. Problem of broadcast video streams encoded in scalable manner to enable heterogeneous mobile devices to render the most appropriate video sub-streams. Due to more than one layer channel switching delay and energy saving problem occur. For this purpose, we proposed a new video broadcast scheme for $3 \mathrm{G}$ mobile devices, where every layer has two parts for every TV channel, two part of every layer takes approximate half time to switch the next TV channel as compare to current broadcast scheme and reduce energy consumption. For the purpose of channel switching, we insert bootstrap in first part of every TV channel. Bootstrap is use to reduce the channel switching delay. Our extensive results confirm that the proposed schemes enable energy saving $0.0065 \%$ observed and achieve less delay $129.0029 \mathrm{msec}$ is possible with typical system parameters as compare to current broadcast scheme.
\end{abstract}

\section{General Terms}

This paper is useful for the scalable video broadcasting to heterogeneous mobile devices. During the classification of this material comes into video broadcast in $3 \mathrm{G}$ mobile devices.

\section{Keywords}

DVB; Digital Video Broadcasting-Handheld (DVB-H), time slicing, handheld terminals; orthogonal frequency division multiplexing (OFDM); multiprotocol encapsulation-forward error correction (MPE-FEC).

\section{INTRODUCTION}

Digital Video Broadcasting (DVB) standard is design for the mobile television. Satellite is a way to transmit several DVB signal. One of them is Digital Video Broadcasting-Handheld (DVB-H) that is most important for multimedia services like television can be received with mobile devices. It is a challenging opportunity to achieving fast data rates in wireless networks and power-limitation in mobile devices for the purpose of efficient use of bandwidth used single frequency networks (SFN) [1-6]

The meaning of the mobile television is television watched on a small mobile device. It may be a paid service that broadcast by the $3 \mathrm{G}$ cellular network (fixed network) to the mobile devices or mobile handheld devices. A base station handles all the mobile devices that receive signal by base station controller. Mobile television is one of the features provided by the $3 \mathrm{G}$ network services. South Korea in 2005 became the first country in the world to have mobile TV. Today, South Korea and Japan are developing sector for the mobile devices.
Mobile Device services were launched by the operator CSL in Hong Kong, March 2006, on the 3G network. Also in 2006 Germany, Italy and US also introduce our 3G network services for mobile television [2]

In 1998 project started for commercial terrestrial digital television services in Europe. After that in 2000, research motivate for the television in mobile devices is possible by DVB-T sponsored by EU. After that EU-sponsored Multimedia Car Platform (MCP) project in 2002 for the receiver to access DVB-T signals broadcast for fixed receivers. Later in 2007 Singapore and Germany cities see that DVB-T shows sufficient flexibility for the mobile devices [2] [3].

DVB-H for instance addresses the energy consumption issues of its predecessor DVB-T by introducing features such as a burst transmission in order to compensate for the smallness and the limited battery capacity of the receivers. This burst technology - together with other factors - increases the time needed for the user to switch between several TV channels by a large amount. Also, when streaming mobile television over UMTS based networks, the limited network bandwidth might prolong the time to display a video stream correctly. Since channel switching is almost inevitable when the user does not know which program they want to see, longer channel switching times lead to a poor quality of experience.

\section{RELATED WORK}

The mobile devices energy saving has been studied for mobile TV networks, where TV channels are coded in non-scalable fashion. The authors of [8] and [9] achieved the energy saving by time slicing. Here time slicing enables mobile devices to turn off their radio components for a significant fraction of time. They, however, did not propose broadcast schemes: they only compute the achieved energy saving for predetermined bursts. Balaguer et al. [10] propose an energy saving strategy by not receiving more forward error correction (FEC) bytes once the data can be successfully reconstructed. In this way, mobile devices can turn off their radio components earlier, and achieve additional energy saving compared to receiving all FEC bytes. Zhang et al. [11] consider mobile devices with an auxiliary short range wireless interface and construct a cooperative network over this short range network to share the IP packets received from the broadcast network. Assuming transmitting data over the short range network is more energy efficient than receiving data from mobile TV networks, this cooperative strategy can save energy. The proposals in [10], [11] are orthogonal and complementary to our work, as they reside in the mobile devices themselves and try to achieve additional energy saving on top of that achieved by time slicing. In contrast, we propose broadcast schemes that are implemented in the base station. 
In previous works authors study the burst transmission problems and propose for mobile TV networks time slicing scheme that broadcast non-scalable video streams to a single class of mobile devices [17], [18], [12]. Here we assume that all mobile devices have the same capability. We consider heterogeneous mobile devices that can only (or opt to) receive parts of video streams in [13], [14], That is, these works assume the scalable video streams are coded into several layers with the same bit rate. However, such assumption may limit the applicability of our preliminary solutions in [13], [14]. For example, uniform bit rates may prevent the video coders from optimally allocating bit rate among layers for higher coding efficiency, and may result in scalable video streams that do not properly match the capability of mobile devices. To address these shortcomings, we propose new broadcast schemes in this paper, which are general in the sense that layers can take any arbitrary bit rates.

\section{PROBLEM STATEMENT}

The scalable video broadcasting problem in mobile TV broadcast networks, where each TV channel is encoded into a scalable video stream with multiple layers, and several TV channels are concurrently broadcast over a shared air medium to many mobile devices with heterogeneous resources. If a user play TV channel 1 then first it will receive complete layer because every layer has a burst and then it can switch to next TV channel. So the channel switching delay and energy saving problem occur. Our goal is to reduce channel switching delay in $3 \mathrm{G}$ mobile $\mathrm{TV}$ broadcasting and also reduce the energy consumption in $3 \mathrm{G}$ mobile devices.

\section{CURRENT SYSTEMS}

There are three main components: video server, IP encapsulator and modulator in video broadcasting from base station, as illustrated in Fig. 1 [1]. The video server encapsulates video data, pre-encoded or lives, into RTP packets, and sends these packets over an IP network to the IP encapsulator. The IP encapsulator receives these IP packets and puts them in MPE (multiprotocol encapsulation) frames.

IP encapsulator use MPE frames for preparing transmission bursts of a specific TV channel, and each MPE frame is sent as one burst. The MPE frame can optionally be protected by Reed-Solomon (R-S) codes. This is achieved by extending the MPE frame into the MPE-FEC frame, which consists of FEC parity bytes. Since mobile devices are vulnerable to bad radio channel conditions, MPE-FEC frames are important as they provide better error resilience [1].

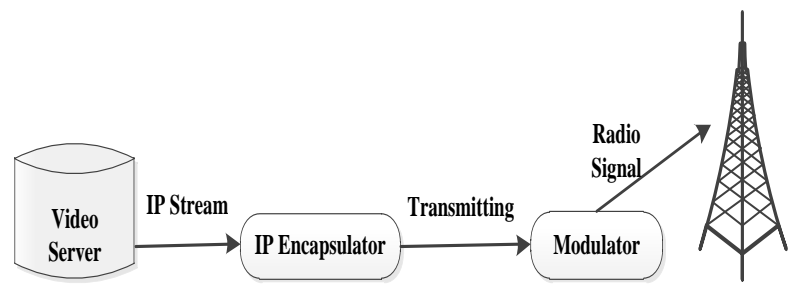

Figure 1: Main Component of Broadcasting

Fig. 2 shows the structure of an MPE-FEC frame [7], divided into two parts: one is application data table (ADT) for carries the IP packets and another R-S (Reed-Solomon) data table (RDT) for carries the parity bytes. Received IP packets are sequentially column-by-column from left to right placed in the ADT. Zeros are padded in the remaining space of the ADT. At the ADT is full (by data and/or zeros), row-by-row arrange all the parity bytes, and stored in the RDT. After computing the parity bytes, the whole MPE-FEC frame is sent, columnby-column, as a burst.

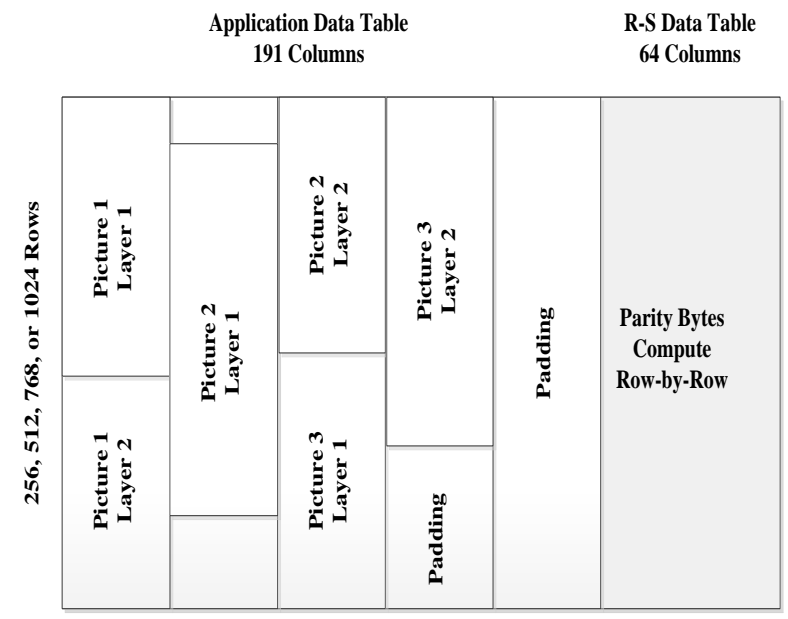

Figure 2: Structure of MPE-FEC frame

Media-FLO from the base station prepare burst for transmission in time-slicing manner [16]. More specifically, Media-FLO base stations transmit signals inform of a superframe structure, where each super-frame have four frames parts and each frame has encoded with 250 OFDM symbols. Several OFDM symbols in every frame has assign for a particular TV channel, and this assignment defines a period of time that mobile devices must turn on their radio components to receive data. Therefore, Media-FLO base stations prepare transmission bursts in the form of assigning OFDM symbols to individual TV channels [1].

\subsection{Single Service (SS)}

In case of single service of video broadcasting network operator for the video server to support scalable video streams can upgraded for heterogeneous mobile devices. That is, each scalable stream is transmitted as a TV channel, and we refer to this scheme as single service (SS). In the SS scheme, existing broadcast systems can be used to broadcast scalable video streams [1]. Unfortunately, this "patched" base station is not efficient as we show in the following illustrative example.

We consider a example for the small time window of three pictures, where each picture is coded into two layers. Here, the author assume that each layer is encapsulated into a single IP packet, and these IP packets are broadcasting by the video server and received by the IP encapsulator in the following order: (picture 1, layer 1), (picture 1, layer 2), (picture 2, layer 1), (picture 2, layer 2), (picture 3, layer 1), (picture 3, layer 2). With the help of IP encapsulator sequentially places IP packets in the receiving order within the first (ADT) part of MPE-FEC frames, these packets are stored into a frame as illustrated in Fig. 2. Then the MPE-FEC frame is sent over the broadcast network as burst broadcasting. Take a mobile device that can only play with base layer (layer 1), this mobile device must receive and process the complete burst (layer1, layer2) for two reasons. First, base layer (layer 1) represents IP packets are scattered all over the MPE-FEC frame, and a deep inspection is required to locate them. Second, IP packets belonging to the parity bytes from various layers, and are useless if some IP packets are not received. Receiving complete bursts degrades the energy saving of that mobile device, because it has to open the radio component for a longer time period to receive some data [1].

\subsection{Parallel Services (PS)}

No additional energy saving in the SS scheme for mobile devices that cannot render the complete video streams. With 
the help of this issue, a better IP encapsulator design needed to author that takes the layering structure of scalable streams into considerations when preparing transmission bursts. In parallel service send each layer of a particular TV channel as a parallel, which can be implemented using multiple IP streams on different multicast IP addresses with time-slicing principle, or using multiple parallel elementary streams [9].

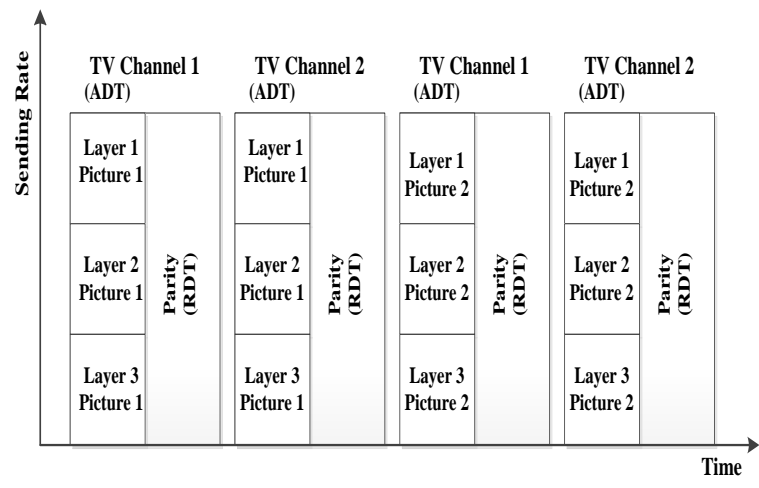

Figure 3: Parallel Service

In fig. 3 video broadcasting of two TV channel serially and every TV channel is encoded with three layers, where each picture have same length (playing time) in a burst. So here with the same length playing data of all three layers containing by a burst and here only one layer is use-full for a particular mobile device, other both layer is receive with one important layer and it takes too much time and it again wait same type for the playing any particular TV channel. Therefore, all mobile devices achieve the same energy saving despite how many layers they receive and decode.

Author observes that parallel service is not so efficient because mobile devices may receive and discard data (in irrelevant layers) that are useless to them. This takes too much longer time of radio components, and thus higher energy saving consumption.

\subsection{Layer-Aware FEC (LAF)}

Layer-Aware FEC allows mobile devices to do encode the complete scalable streams to turn off their radio components before each burst ends. If we again use the illustrative example of SS, the burst packets should be sent in the following order: (picture 1, layer 1), (picture 2, layer 1), (picture 3, layer 1), (picture 1, layer 2), (picture 2, layer 2), (picture 3, layer 2), as illustrated in Fig. 4. The layer number can be pre-pended before the MPE header as an one-byte extension header, which allows mobile devices to efficiently determine the boundaries between layers, e.g., between (picture 3, layer 1) and (picture 1, layer 2).

However, even after reordering IP packets, mobile devices still have to receive complete bursts in order to perform error correction, which again prevents them from getting higher energy saving [1]. This is because the FEC parity bytes are sent after all the IP packets, at the end of each burst. To address this issue, we can compute parity bytes column-bycolumn, and send these bytes right after each column of data bytes. This allows mobile devices to perform error corrections without receiving complete bursts. That is, mobile devices can receive partial bursts and turn off the radio components to save energy. We call this new frame format as Layer-Aware FEC (LAF) frame, which is illustrated in Fig. 4.

While LAF frame allows mobile devices to efficiently receive and extract sub-streams, it has several drawbacks. First, LAF does not comply with mobile TV standards, which causes compatibility issues between the base station and mobile devices. Second, implementing LAF requires significant changes as error corrections are usually done in hardware/firmware for the sake of performance. Third, computing parity bytes column-by-column makes the FEC decoder vulnerable to bursty channel errors because it does not provide virtual time interleaving as by MPE-FEC frames [15]. Most importantly, LAF scheme achieves lower energy saving compared to the scheme developed in that section

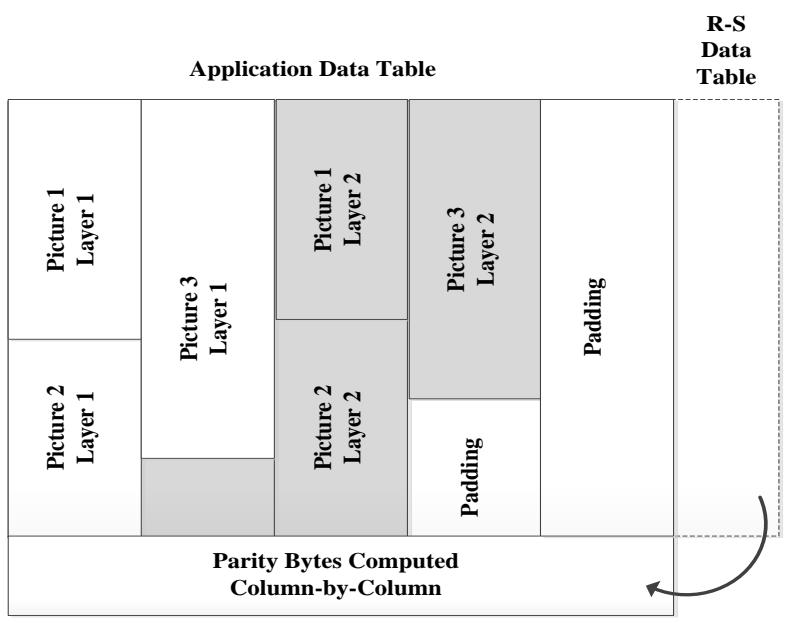

Figure 4: Layer-Aware FEC (LAF)

\subsection{Summary}

SS, PS, LAF these are three schemes to broadcast scalable video streams in current mobile TV networks. The SS and PS schemes require all mobile devices, despite which classes they are in, to receive complete scalable streams. Therefore, they result in no energy saving differentiation as all mobile devices turn on their radio components for the same amount of time. While the LAF scheme enables energy saving differentiation, it is [1]:

1. Not standard compliant,

2. Hard to implement,

3. Vulnerable to bad channel conditions, and

4. Achieves lower energy saving.

Hence, we conclude that current mobile TV broadcast networks cannot efficiently support scalable video streams, and we need to design better broadcast schemes to solve Problem.

\section{PROPOSE SCHEME 5.1 Propose Scheme}

We propose a new broadcast scheme that is useful to achieve energy saving diversity without incurring long channel switching delays, and this propose scheme also useful to reduce the burst packet time duration.

Our proposed broadcast scheme aims to reduce channel switching delays in mobile television systems, channel switching from one channel to another channel require an indefinite amount of time. In our proposed video broadcasting scheme, we send a layer into two burst. Two burst will be helpful to reduce the delay and energy consumption. Every first burst of every layer will be attached with bootstrap to reduce the channel switching delay. With the two burst of 
every layer bootstrap memory size will also reduce because bootstrap size decide on time taking by the burst to receive.

Fig. 6 illustrates how the bootstrap inserts between bursts first parts of different TV channel. Here every layer has two parts for every TV channel and every first part of every TV channel attach with bootstrap. This figure shows that two bootstrap bursts for TV channel 1 and 2, respectively, are added between any two first parts of every burst layer. Since the bootstrap bursts are sent very often, the user who switches to a new channel can receive the bootstrap bursts without receiving the layer 1 of previous TV channel and start playing the base layer very quickly. In our proposed broadcast scheme burst size is just half with comparison to the current broadcast scheme, so it will take half time to receive by user. If user want to see TV channel 1, it can find to see it in fraction of seconds and play with it, if he/she want to continuous then no problem, this channel will play continuously, otherwise user can switch to the TV channel 2 and for that channel switching delay here we already add a bootstrap between two first part of every burst layer. Due to the just half of every burst size bootstrap will play only for that time in which the burst packet will be receive by user and after that receive packet will start to play.

\subsection{Burst Parameters}

To evaluate the proposed scheme $\mathrm{T}_{\mathrm{ON}}, \mathrm{T}_{\mathrm{OFF}}$, delay and energy consumption parameters could be defined freely, but offcourse, some are implied already. For example, the constant or average data bit-rate is known before burst scheduling. Then, the burst time can be calculated by the size and the bitrate of each burst. Where, BS (burst size), BBR (burst bit rate) and $\mathrm{OH}$ (overhead) in equation (1):

$$
\mathrm{T}_{\mathrm{ON}}=\left(\frac{\mathrm{BS}}{(\mathrm{BBR} \times \mathrm{OH})}\right)+\mathrm{T}_{\text {Sync }}
$$

The Off-Time will be calculated by the burst bit-rate and the average or constant video bit-rate, and adding the On-Time. Where, BS (burst size), BBR (burst bit rate) and CBR (constant bit rate) in equation (2):

$$
\mathrm{T}_{\mathrm{OFF}}=\left(\frac{\mathrm{BBR}}{\mathrm{CBR}}\right)-\mathrm{T}_{\mathrm{ON}}
$$

Overhead is a factor that compensates for the additional headers included in the MPEG-2 Transport Stream. It should be set to 0.96 . TSync is the synchronization time needed for the receiving unit to tune into the DVB signal again. Its value can change depending on the device used and lies in the range from $80 \mathrm{~ms}$ to $200 \mathrm{~ms}$ [19-20].

The energy saving potential in percentage can be calculated. Where, CBR (constant bit rate), BBR (burst bit rate), $\mathrm{OH}$ (overhead) and BS (burst size) in the equation (3):

$$
\mathrm{P}=\left(1-\mathrm{CBR} \times\left(\frac{1}{\mathrm{BBR} \times \mathrm{OH}}+\frac{\mathrm{T}_{\text {sync }}}{\mathrm{BS}}\right)\right) \times 100 \%
$$

\subsection{Calculating Delay}

In a simplified model, only On and Off times derived from the parameters stated before are considered for calculating delay. In fact, there are more factors possibly increasing the delay.

Then, the absolute minimum delay would be equal to TON. The worst case scenario results in the receiving unit starting immediately after the beginning of a desired burst the waiting time is as long as $2 . \mathrm{T}_{\mathrm{ON}}+\mathrm{T}_{\mathrm{OFF}}$. However, in weak reception conditions, frame losses lead to much longer delays than mentioned, with each lost frame resulting in an additional delay of $\mathrm{T}_{\mathrm{ON}}+\mathrm{T}_{\mathrm{OFF}}$ [20]. This is not considered here.

The average delay and variable delay always varied, that can be calculated by these equations (4) and (5):

Average delay $(\mathrm{D})=\frac{3 \cdot \mathrm{T}_{\mathrm{ON}}+\mathrm{T}_{\mathrm{OFF}}}{2}$

Variable delay $(\mathrm{D})=\frac{\left(\mathrm{T}_{\mathrm{ON}}+\mathrm{T}_{\mathrm{OFF}}\right)^{2}}{12}$

The total delay in a channel for a single layer is addition of average delay and variable delay, that can be calculated by the equation (6):

$\mathrm{D}=($ Average delay + Variable Delay $)$

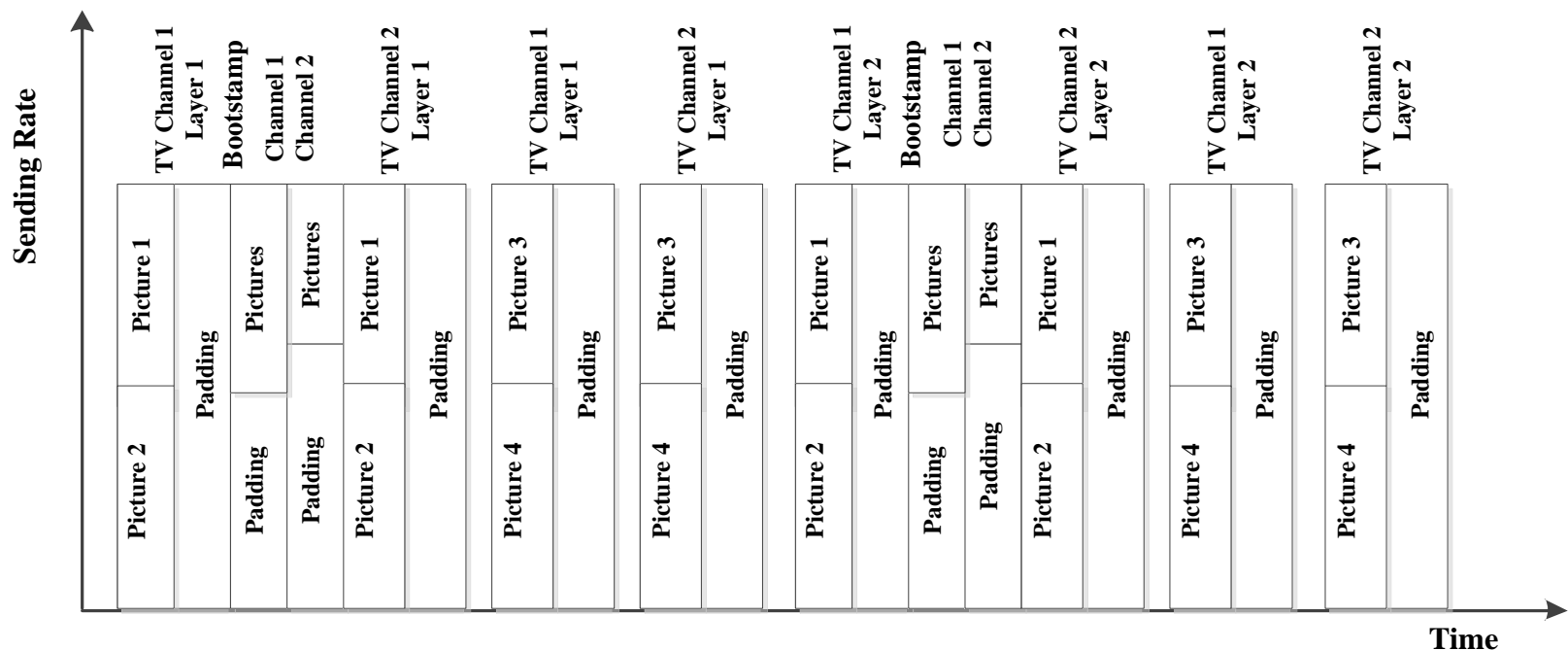

Fig 6: Proposed Scheme to Broadcast TV Channels 


\section{RESULTS}

There are two TV channel broadcast schemes (current broadcast scheme and proposed broadcast scheme) that show delay (msec) and energy saving in percentage. Authors [1920] proposed some mathematical formula to calculate the TON, TOFF, Variable delay, Average delay, Delay and Energy saving. By the help of these formulas, we simulate both TV channel broadcast schemes in MATLAB. These MATLAB values show that we have less delay 129.0029 msec and more energy saving $0.0065 \%$. So, these values proved that our proposed broadcast scheme is much better than current broadcast scheme.

\subsection{Delay on Different Burst Size}

In fig 7 show the effect in delay (msec) on different-different burst size $\mathrm{b}$ from $500 \mathrm{~kb}$ to $3000 \mathrm{~kb}$ but according to DVB-H standard [9] maximum burst size should not more than 1565 $\mathrm{kb}$. In broadcasting scheme after $1500 \mathrm{~kb}$ burst size, burst will transfer but non-standard according to DVB-H. Figure shows that our proposed broadcast scheme have less delay (129.0029 $\mathrm{msec}$ ) with comparison to current broadcast scheme.

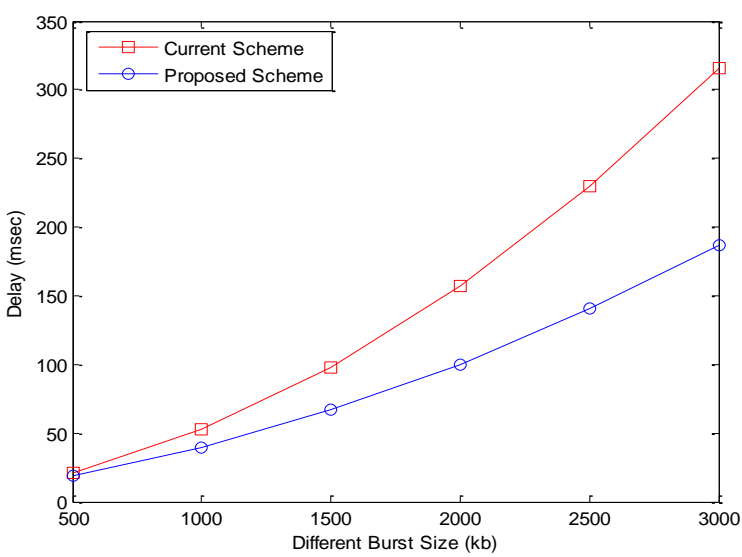

Fig 7: Effect of Delay on different bursts size

\subsection{Energy Saving on Different Burst Size}

In fig 8 show the effect of energy saving in percentage on different-different burst size b from $500 \mathrm{~kb}$ to $3000 \mathrm{~kb}$ respectively. Figure shows that our proposed broadcast scheme has more energy efficient $(0.0065 \%)$ with comparison to current broadcast scheme.

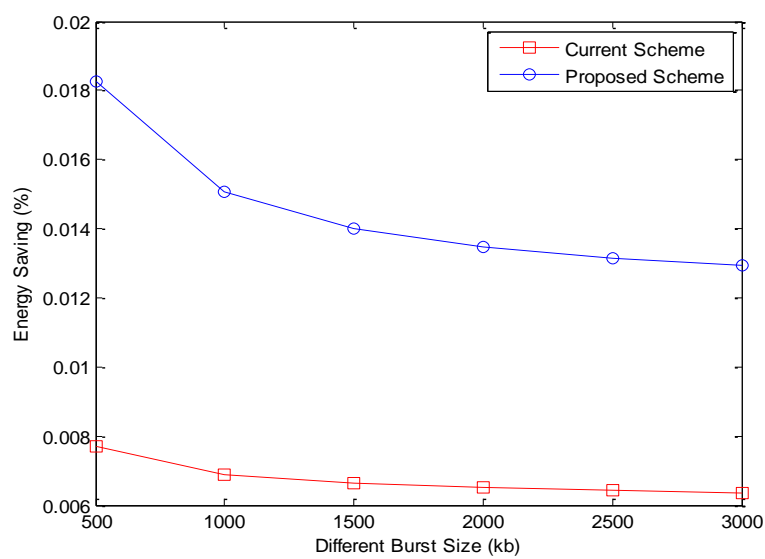

Fig 8: Effect of Energy saving on different bursts size

\section{CONCLUSION}

In this paper, we studied the problem of broadcasting scalable video streams over a shared air medium to mobile devices with heterogeneous resources; such that mobile devices can render the most appropriate sub-streams while achieving high energy saving and low channel switching delay. We analyzed current mobile TV networks, and showed that they cannot efficiently broadcast scalable coded streams. Then we proposed a scalable broadcast scheme. With the help of mathematical formulation correctness proved of our proposed broadcast scheme, and we analytically quantified their performance in terms of energy saving and delay. Proposed broadcast scheme is implemented in MATLAB with some mathematical formulation to show their practicality and efficiency. We apply proposed broadcast scheme on mobile TV channel find less delay $129.0029 \mathrm{msec}$ and also find more energy saving $0.0065 \%$.

\section{REFERENCES}

[1] Cheng-Hsin Hsu, Mohamed Hefeeda, "Flexible Broadcasting of Scalable Video Streams to Heterogeneous Mobile Devices," IEEE Transaction on Mobile Computing, vol. 10, no. 3, March 2011.

[2] G. Faria, J. Henriksson, et. al., "DVB-H: Digital broadcast services to handheld devices," Proc. of the IEEE, vol. 94, no. 1, pp. 194-209, January 2006.

[3] ETSI, Digital Video Broadcasting (DVB); "Transmission System for Handheld Terminals (DVB-H)," EN 302-304 V1.1.1, (2004-2011).

[4] ETSI, Digital Video Broadcasting (DVB); "DVB Specification for Data Broadcasting," EN 301-192 v1.4.1, November 2004.

[5] ETSI, Digital Video Broadcasting (DVB); "Specification for Service Information (SI) in DVB systems," EN 300468 V1.11.1, (2010-04).

[6] ETSI, Digital Video Broadcasting (DVB); "DVB-H Implementation Guidelines," TR 102-377 V1.3.1, (200903).

[7] C. Hellge, T. Schierl, et. al., "Mobile TV using scalable video coding and layer-aware forward error correction," in Proc. of IEEE International Conference on Multimedia and Expo (ICME’08), Hannover, Germany, April 2008, pp. 1177-1180.

[8] X. Yang, Y. Song, et. al., "Performance Analysis of Time Slicing in DVB-H," Proc. Joint IST Workshop Mobile Future and Symp. Trends in Comm. (SympoTIC '04), pp. 183-186, Oct. 2004.

[9] ETSI, Digital Video Broadcasting (DVB); "DVB-H Implementation Guidelines," EN 102-377 Ver. 1.3.1, May 2007.

[10] E. Balaguer, F. Fitzek, et. al., "Performance Evaluation of Power Saving Strategies for DVB-H Services Using Indoor and Mobile Radio Comm. (PIMRC '05), pp. 2221-2226, Sept. 2005.

[11] Q. Zhang, F. Fitzek, and M. Katz, "Cooperative Power Saving Strategies for IP-Services Supported over DVB-H Networks," Proc. IEEE Wireless Comm. and Networking Conf. (WCNC '07), pp. 4107- 4111, Mar. 2007.

[12] C. Hsu, M. Hefeeda, "Bounding Switching Delay in Mobile TV Broadcast Networks," Proc. ACM/SPIE 
Multimedia Computing and Networking (MMCN '09), pp. 72530A:1-72530A:12, Jan. 2009.

[13] C. Hsu, M. Hefeeda, "Video Broadcasting to Heterogeneous Mobile Devices," Proc. Int'l Conf. IFIP Networking, pp. 600-613, May 2009.

[14] C. Hsu, M. Hefeeda, "Multi-Layer Video Broadcasting with Low Channel Switching Delays," Proc. IEEE Int'l Packet Video Workshop (PV '09), May 2009.

[15] G. Faria, J. Henriksson, et. al., "DVB-H: Digital Broadcast Services to Handheld Devices," Proc. IEEE, Vol. 94, No. 1, pp. 194-209, Jan. 2006.

[16] K. Daoud, "Performance Comparison of the DVB-H and FLO Mobile Broadcasting Systems," Proc. IEEE Int'1 Symp. Consumer Electronics (ISCE '07), pp. 1-7, June 2007.
[17] C. Hsu, M. Hefeeda, "Broadcasting Video Streams Encoded with Arbitrary Bit Rates in Energy-Constrained Mobile TV Networks," IEEE/ACM Trans. Networking, Vol. 18, No. 3, pp. 681- 694, June 2010.

[18] M. Hefeeda, C. Hsu, "On Burst Transmission Scheduling in Mobile TV Broadcast Networks," IEEE/ACM Trans. Networking, Vol. 18, No. 2, pp. 610-623, Apr. 2010.

[19] M. Rezaei, I. Bouazizi, V. Vadakital et. al., "Optimal Channel Changing Delay for Mobile TV Over DVB-H," May 2007, pp. 1-5.

[20] ETSI, Digital Video Broadcasting (DVB); "DVB-H Implementation Guidelines," EN 102-377, Ver.1.2.1, May 2007. 